Recepción: 08 / 09 / 2016

Aceptación: 23 / 01 / 2017

Publicación: 06 / 03 / 2017

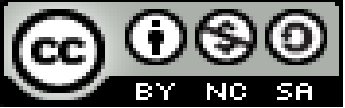

Ciencias económicas y empresariales

Artículo de investigación

\title{
Herramientas de análisis para definir estrategias empresariales
}

\author{
Analysis tools to define business strategies \\ Ferramentas de análise para definir as estratégias de negócios \\ Hernán O. Arellano-Díaz ${ }^{1}$ \\ harellano@espoch.edu.ec
}

Correspondencia: harellano@espoch.edu.ec

\footnotetext{
I Master en Dirección de Empresas, Diplomado en Estadística Informática Aplicada a la Educación, Diplomado Superior en Inteligencia Emocional y Desarrollo del Pensamiento, Especialista en Computación Aplicada al Ejercicio Docente, Ingeniero de Empresas, Docente de la Escuela Politécnica de Chimborazo ESPOCH, Riobamba, Ecuador.
} 


\title{
Resumen
}

La delimitación de estrategias adecuadas para una empresa, supone partir de un estudio para determinar sus particularidades, luego de un diagnóstico general. Un plan de mercadotecnia conveniente, respalda tareas como planear, establecer metas, promover y distribuir los bienes y servicios en aras de satisfacer deseos y necesidades de los clientes. Este artículo, pone énfasis en tres de las principales herramientas de análisis del entorno empresarial, para facilitar el diseño de adecuadas estrategias de mercadotecnia en consonancia con las particularidades de cada empresa. Palabras clave: estrategias empresariales; entorno empresarial; mercadotecnia.

\begin{abstract}
The delimitation of strategies suitable for a company, involves starting a study to determine their particularities, after a general diagnosis. A convenient marketing plan supports tasks such as planning, setting goals, promoting and distributing goods and services in order to satisfy customers' wishes and needs. This article emphasizes three of the main analysis tools of the business environment, to facilitate the design of adequate marketing strategies in line with the particularities of each company.
\end{abstract}

Keywords: business strategies; business environment; marketing.

\section{Resumo}

O delineamento de estratégias apropriadas para um negócio, é baseado em um estudo para determinar suas características após um diagnóstico geral. Plano de marketing conveniente, suporta tarefas como planejamento, estabelecimento de metas, promover e distribuir produtos e serviços, a fim de satisfazer os desejos e necessidades dos clientes. Este artigo enfatiza três principais ferramentas de análise do ambiente de negócios para facilitar o desenho de estratégias de marketing adequadas, de acordo com as particularidades de cada empresa.

Palavras chave: estratégias de negócios; ambiente de negócios; marketing. 


\section{Introducción}

Una de las áreas más importantes dentro de la administración de empresas, lo constituye la mercadotecnia, desde la cual la gerencia puede garantizar el adecuado posicionamiento de la empresa. Un apropiado plan de mercadotecnia permite planear, establecer metas, promover y distribuir los bienes y servicios para satisfacer deseos y necesidades de los clientes presentes y potenciales.

Fischer \& Espejo (2004:6), definen a la mercadotecnia como: "Aquella actividad humana dirigida a satisfacer necesidades, carencias y deseos a través de procesos de intercambio." La mercadotecnia centra su objetivo en determinar los intereses de los clientes, de manera que se puedan ofrecer los productos o servicios que satisfagan sus necesidades.

Mediante la investigación de mercados se determinan aspectos referentes a la situación actual del producto, de la competencia, y de las preferencias y necesidades de los clientes. Este estudio deriva hacia la empresa, esencialmente, un plan general que orienta y regula todas las políticas y acciones de ésta para el negocio que desarrolla, bien sea para un futuro de corto, mediano o largo plazo.

El estudio de la orientación hacia el mercado, requiere de una investigación previa de aquellos factores que inciden en la calidad o eficiencia de los productos o servicios que ofrece la empresa. Determinar estos elementos permite el establecimiento de estrategias adecuadas que impulsen el posicionamiento empresarial (Kootnz H. \& Weihrich, 1998).

La determinación de estrategias adecuadas para una empresa, debe partir de un análisis que permita delimitar las particularidades de esta a partir de un diagnóstico general. Otro aspecto de gran importancia, es la evaluación de la situación externa, representada por el mercado y los competidores; así como la situación interna, es decir, las debilidades y fortalezas de las empresas.

Este análisis del mercado puede resultar vital para las empresas, ya que permitirá descubrir las oportunidades y amenazas del entorno y el mercado, así como las debilidades y fortalezas de la propia empresa. 
El presente trabajo se propone profundizar en tres de las principales herramientas de análisis del entorno empresarial, que facilitan el diseño de adecuadas estrategias de mercadotecnia acorde a las particularidades de cada empresa. Esto posibilitará que las empresas logren, desde su planeación estratégica, resultados que generen, además de ganancias, valor socioeconómico. Es decir, no sólo les permitirá ganar dinero con la venta de sus productos/servicios, sino que favorecerá además el posicionamiento de sus marcas comerciales y de la imagen de la empresa.

\section{Desarrollo}

El plan de mercadotecnia funciona como instrumento que ayuda a dirigir y coordinar actividades que debe realizar una empresa para lograr un objetivo específico. En este objetivo, se detalla qué es lo que se desea lograr, de forma clara y cuantificable. Las diferentes actividades que incluyen el plan, se contemplan a corto, mediano y largo plazo en base al análisis de la empresa frente a su mercado meta actual y potencial (Cohen, 2004).

Fischer y Espejo lo definen como un programa que incluye una estructura y una serie de actividades que deberán realizarse para alcanzar un objetivo determinado (Fischer y Espejo, 2004:36). Para la implementación de un plan de mercadotecnia se debe realizar un análisis donde se conozcan aquellos factores, tanto internos como externos que relacionarán las actividades a realizar dentro de la empresa en el desarrollo de este plan y en un segundo momento determinar las particularidades de la empresa a partir de las fortalezas y debilidades que la misma tiene.

Para la realización de este análisis, existen tres herramientas fundamentales, que la gerencia empresarial debe dominar en aras de lograr una evaluación certera de su entorno y en base a esta determinar las estrategias más adecuadas para lograr elevar el posicionamiento de la empresa en el mercado. Estas herramientas son: la matriz de evaluación de los factores internos (EFI), la matriz de evaluación de los factores externos (EFE) y la matriz FODA.

\section{La matriz de evaluación de los factores internos (EFI)}

Un paso certero para realizar una auditoría interna de la administración estratégica en una empresa consiste en constituir una matriz EFI. Este instrumento resulta de gran valía para formular estrategias, ya que resume y evalúa las fuerzas y debilidades más importantes dentro de 
las áreas funcionales de un negocio y además ofrece una base para identificar y evaluar las relaciones entre dichas áreas.

Al elaborar una matriz EFI es necesario aplicar juicios intuitivos, por lo que el hecho de que esta técnica tenga apariencia de un enfoque científico no se debe interpretar como si la misma fuera del todo contundente. Es bastante más importante entender a fondo los factores incluidos que las cifras reales.

Los pasos para realizar un análisis del entorno empresarial desde la matriz EFI son los siguientes:

1. Realizar una lista de los factores de éxito identificados mediante el proceso de la auditoría interna. Para esto es preciso usar entre diez y veinte factores internos en total, que incluyan tanto fuerzas como debilidades. Primero se deben considerar las fuerzas y después las debilidades. En este primer paso es importante ser lo más específico posible y usar porcentajes, razones y cifras comparativas.

2. Asignar una puntuación entre 0.0 (no importante) a 1.0 (absolutamente importante) a cada uno de los factores. El puntaje adjudicado a un factor dado indica la importancia relativa del mismo para alcanzar el éxito de la empresa. Independientemente de que el factor clave represente una fuerza o una debilidad interna, los factores que se consideren que repercutirán mas en el desempeño de la organización deben llevar la puntuación más alta. El total de todos los puntos debe de sumar1.0.

3. Asignar una calificación entre 1 y 4 a cada uno de los factores a efecto de indicar si el factor representa una debilidad mayor (calificación $=1$ ), una debilidad menor (calificación $=2$ ), una fuerza menor (calificación =3) o una fuerza mayor (calificación =4). Así, las calificaciones se refieren a la compañía, mientras que los puntos del paso 2 se refieren a la industria.

4. Multiplicar el punto de cada factor por su calificación correspondiente para determinar una calificación ponderada para cada variable. 
5. Sumar las calificaciones ponderadas de cada variable para determinar el total ponderado de la organización entera.

Sea cual fuere la cantidad de factores que se incluyen en una matriz EFI, el total ponderado puede ir de un mínimo de 1.0 a un máximo de 4.0, siendo la calificación promedio de 2.5. Los totales ponderados muy por debajo de 2.5 caracterizan a las organizaciones que son débiles en lo interno, mientras que las calificaciones muy por arriba de 2.5 indican una posición interna fuerza.

Cuando un factor interno clave es una fuerza y al mismo tiempo una debilidad, el factor debe ser incluido dos veces en la matriz EFI y a cada uno se le debe asignar tanto un punto como una calificación.

\section{La matriz de evaluación de los factores externos (EFE)}

La matriz de evaluación de los factores externos (EFE) permite a los estrategas resumir y evaluar información económica, social, cultural, demográfica, ambiental, política, gubernamental, jurídica, tecnológica y competitiva del entorno empresarial. La elaboración de una Matriz EFE consta de cinco pasos:

1. Realizar una lista de los factores críticos o determinantes para el éxito identificados en el proceso de la auditoría externa. Es importante destacar un total de entre diez y veinte factores, incluyendo tanto oportunidades como amenazas que afectan a la empresa y su industria.

En esta lista, primero se debe tomar en consideración las oportunidades y después las amenazas. En la determinación de cada uno de los factores a señalar es necesario ser lo más específico posible, usando porcentajes, razones y cifras comparativas en la medida de lo posible.

2. Debe asignarse un punto relativo a cada factor, de 0.0 (no es importante) a 1.0 (muy importante). El punto indica la importancia relativa que tiene ese factor para alcanzar el éxito en la industria de la empresa. Las oportunidades suelen tener puntos más altos que las amenazas, pero éstas, a su vez, pueden tener puntajes altos si son especialmente graves o amenazadoras. Los pesos adecuados se pueden determinar comparando a los competidores que tienen éxito con los 
que no lo tienen o analizando el factor en grupo y llegando a un consenso. La suma de todos los puntos asignados a los factores debe sumar 1.0.

3. Una vez establecida la puntuación a cada uno de los factores identificados, ya sea como fortaleza o amenaza, se debe asignar una calificación de 1 a 4 a cada uno de los factores determinantes para el éxito con el objeto de indicar si las estrategias presentes de la empresa están respondiendo con eficacia al factor.

Esta calificación que se asigna se establece por el siguiente patrón: 4 = una respuesta superior, 3 = una respuesta superior a la media, $2=$ una respuesta media y $1=$ una respuesta mala. Las calificaciones se basan en la eficacia de las estrategias de la empresa. Así pues, las calificaciones se basan en la empresa, mientras que los puntos del paso 2 se basan en la industria.

4. Una vez establecidas las calificaciones, se debe multiplicar el punto de cada factor por su calificación para obtener una calificación ponderada.

5. Posteriormente se suman las calificaciones ponderadas de cada una de las variables para determinar el total ponderado de la organización.

Independientemente de la cantidad de oportunidades y amenazas clave incluidas en la matriz EFE, el total ponderado más alto que puede obtener la organización es 4.0 y el total ponderado más bajo posible es 1.0. El valor del promedio ponderado es 2.5. Un promedio ponderado de 4.0 indica que la organización está respondiendo de manera excelente a las oportunidades y amenazas existentes en su industria. Es decir, que las estrategias de la empresa están aprovechando con eficacia las oportunidades existentes y minimizando los posibles efectos negativos de las amenazas externas. Un promedio ponderado de 1.0 indica que las estrategias de la empresa no están capitalizando las oportunidades ni evitando las amenazas externas.

\section{Matriz de evaluación FODA}

El análisis FODA es una herramienta que permite conformar un cuadro de la situación actual del objeto de estudio (empresa, institución u organización, etc.) permitiendo de esta manera obtener 
un diagnóstico preciso que posibilite, en función de ello, tomar decisiones acordes con los objetivos y políticas formulados.

Luego de haber realizado el primer análisis FODA, se aconseja realizar sucesivos análisis de forma periódica teniendo como referencia el primero, con el propósito de conocer si se está cumpliendo con los objetivos planteados en la formulación estratégica. Esto es aconsejable dado que las condiciones externas e internas son dinámicas y algunos factores cambian con el paso del tiempo, mientras que otros sufren modificaciones mínimas.

La frecuencia de estos análisis de actualización dependerá del tipo de objeto de estudio del cual se trate y el contexto en que este se desenvuelve.

En términos del proceso de Marketing en particular, y de la administración de empresas en general, es justo considerar que la matriz FODA es el nexo que permite pasar del análisis de los ambientes interno y externo de la empresa hacia la formulación y selección de estrategias a seguir en el mercado.

El objetivo primario del análisis FODA consiste en obtener conclusiones sobre la forma en que el objeto estudiado será capaz de afrontar los cambios y las turbulencias en el contexto, (oportunidades y amenazas) a partir de sus fortalezas y debilidades internas.

Ese constituye el primer paso esencial para realizar un correcto análisis FODA. Cumplido el mismo, el siguiente consiste en determinar las estrategias a seguir.

Para comenzar un análisis FODA se debe hacer una distinción crucial entre las cuatro variables por separado y determinar qué elementos corresponden a cada una.

A su vez, en cada punto del tiempo en que se realice dicho análisis, resultaría aconsejable no sólo construir la matriz FODA correspondiente al presente, sino también proyectar distintos escenarios de futuro con sus consiguientes matrices FODA y plantear estrategias alternativas. 
Tanto las fortalezas como las debilidades son internas de la organización, por lo que es posible actuar directamente sobre ellas. En cambio, las oportunidades y las amenazas son externas, y solo se puede tener injerencia sobre ellas modificando los aspectos internos.

Las cuatro variables que intervienen en la matriz FODA pueden definirse en el contexto empresarial como:

Fortalezas: son las capacidades especiales con que cuenta la empresa, y que le permite tener una posición privilegiada frente a la competencia. Recursos que se controlan, capacidades y habilidades que se poseen, actividades que se desarrollan positivamente, etc.

Oportunidades: son aquellos factores que resultan positivos, favorables, explotables, que se deben descubrir en el entorno en el que actúa la empresa, y que permiten obtener ventajas competitivas.

Debilidades: son aquellos factores que provocan una posición desfavorable frente a la competencia, recursos de los que se carece, habilidades que no se poseen, actividades que no se desarrollan positivamente, etc.

Amenazas: son aquellas situaciones que provienen del entorno y que pueden llegar a atentar incluso contra la permanencia de la organización.

La importancia de confeccionar y trabajar con una matriz de análisis FODA reside en que este proceso permite buscar y analizar, de forma proactiva y sistemática, todas las variables que intervienen en el negocio con el fin de tener más y mejor información al momento de tomar decisiones.

Si bien la herramienta estratégica ideal para plasmar la misión, la visión, las metas, los objetivos y las estrategias de una empresa es el Plan de Negocios, realizando correctamente el análisis FODA se pueden establecer las estrategias Ofensivas, Defensivas, de Supervivencia y de Reordenamiento necesarias para cumplir con los objetivos empresariales planteados (Kotler, 2001). 
El patrón para realizar un análisis desde la matriz FODA queda establecido de la siguiente manera:

\begin{tabular}{l|l|}
\hline Fortalezas & Debilidades \\
\hline F1- & $\begin{array}{l}\text { Variables estructurales internas de difícil } \\
\text { eliminación o reducción (estrategias a largo } \\
\text { plazo) }\end{array}$ \\
F3- & D- \\
\hline Oportunidades & Amenazas \\
\hline P- & Permanentes \\
(no asociadas a nuestras debilidades)
\end{tabular}

Una vez completada la planilla con las variables correspondientes a cada factor, el paso siguiente es el análisis de las mismas y la preparación de las estrategias de acción correspondiente a la realidad evidenciada.

La forma de presentación más acertada de la formulación de estrategias es la siguiente: 
Estrategias (E):

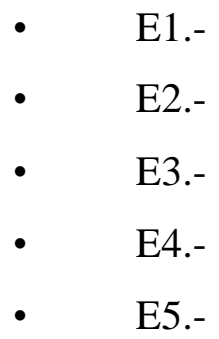

Al momento de escribir las diferentes estrategias se deben colocar las referencias de las variables analizadas en la planilla FODA correspondientes a los factores (fortalezas, debilidades, oportunidades y amenazas)

\section{Conclusiones}

En una época de globalización y de alta competitividad, es cada vez más necesario estar alerta a las exigencias y expectativas del mercado, es de vital importancia para asegurar el éxito de las empresas hacer uso de técnicas y herramientas adecuadas que permitan mejorar el posicionamiento de éstas, y a partir de las cuales se puedan trazar estrategias de desarrollo y planes de mercadotecnia acertadas para cumplir con las expectativas del mercado. Esto conduce a la realización de un conjunto de actividades como lo son un análisis de la competencia, el lugar de venta del servicio, que tanta publicidad existe en el mercado, precios, etc.

Con el diseño y puesta en marcha de estrategias adecuadas y planes de mercadotecnia pertinentes, la empresa contará con un mayor control de los recursos de mercadeo, debido a que podrá realizar una planeación de las actividades con sus respectivos presupuestos y después, realizar un análisis del impacto que estas tuvieron frente a los objetivos planteados.

\section{Referencias bibliográficas}

Kotler, P. \& Armstrong, G. (2001). Segmentación de mercados, mercados meta y posicionamiento para obtener una ventaja competitiva. México: Prentice Hall Hispanoamérica.

Fischer L. \& Espejo J. (2004). Mercadotecnia. México: Mc Graw Hill. 
Cohen, W. A. (2004). Plan de Mercadotecnia. México: Compañía Editorial Continental.

Kootnz H. \& Weihrich. (1998). Administración, una perspectiva global. México: Mc Graw Hill. 